\title{
Distal Biceps Brachii Tendon Transfer for Re-establishing Extrinsic Finger Function: Feasibility Study in Cadavers
}

Matthew D. Welsch, Alexander D. Mih, Brock D. Reite, Gregory A. Merrell

\begin{abstract}
Purpose: To determine the anatomic feasibility of transferring the biceps brachii tendon into either the extensor digitorum communis (EDC) or flexor digitorum profundus (FDP), determine the excursion imparted to EDC and FDP tendons after transfer, and compare the work capacity of the cadaver biceps to previously published data on the biceps as well as the recipient muscles by calculating the physiological cross-sectional area.
\end{abstract}

Methods: Four fresh-frozen cadaver shoulder-elbow-wrist specimens were used to measure tendon excursion that can be obtained with transfer of the distal biceps tendon into either the EDC or FDP. Two cadavers had distal biceps-to-EDC transfer performed, and the other two had distal biceps-to-FDP performed. Passive and active loading of each specimen was then performed to determine tendon excursion. A post-hoc analysis of the physiological crosssectional area (PCSA) of the biceps muscle was then performed on each specimen.

Results: Distal biceps-to-EDC transfer resulted in an average of $24 \mathrm{~mm}$ of tendon excursion with passive loading, and 24 mm of tendon excursion with active loading. Distal biceps-to-FDP transfer resulted in an average of $24 \mathrm{~mm}$ of tendon excursion with passive loading, and $24 \mathrm{~mm}$ of tendon excursion with active loading. The average PCSA was $3.6 \mathrm{~cm}^{2}$.

Conclusions: Transfer of the distal biceps tendon into the EDC or FDP is anatomically feasible and provides roughly $24 \mathrm{~mm}$ of tendon excursion to the tendon units. The PCSA in the

This is the author's manuscript of the article published in final edited form as:

Welsch, M. D., Mih, A. D., Reiter, B. D., \& Merrell, G. A. (2017). Distal Biceps Brachii Tendon Transfer for Reestablishing Extrinsic Finger Function: Feasibility Study in Cadavers. The Journal of hand surgery, 43(3), 290.e1-290.e7. http://dx.doi.org/10.1016/j.jhsa.2017.10.003 
specimens used is slightly lower than other published data; it closely approximates the PCSA of the EDC, but is almost half of the PCSA of the FDP in previously published data.

Clinical Relevance: We describe novel transfer options for restoring finger flexion and extension in patients lacking FDP or EDC function. 


\section{Introduction}

Tendon transfer in the upper extremity has evolved with certain well known tenets. These include transfer through an uninjured or healed surgical bed, optimization of passive motion across the joint that the transfer travels, expendability and voluntary muscle control of the transferred muscle, appropriate line of action of the transferred muscle with or without the establishment of a pulley, and synergism between the transferred muscle original function and its new function. Adhering to these principles increases the effectiveness of the muscle transfer in performing its new action. ${ }^{1}$

Conditions such as Volkmann’s ischemic contracture, brachial plexus palsy, and tetraplegia involve upper extremity dysfunction in which the forearm muscles allowing finger extension and/or flexion are non-functional while muscles of the arm such as brachialis or biceps may be spared in these conditions. These muscles normally traverse the elbow to position the forearm and hand in space and insert in proximity to the origin of muscles controlling finger extension and flexion. This positional relationship may allow for transfer of working muscles to denervated or nonfunctional musculotendinous units to restore function that has been lost. This “redistribution of assets” can provide important clinical improvements in selected patients. ${ }^{1}$

Various methods of restoring function in the hand have been used including reinnervating denervated motor units, transfer of viable and expendable muscle units, and free muscle transfer. There are a limited number of appropriate motor units for transfer, with availability depending on the degree of injury in Volkmann's contracture or brachial plexus injury or level of spinal cord injury in tetraplegia. The possible availability of the biceps for use in reestablishing finger flexion or extension in patients with limited options may be beneficial. 
The purpose of this investigation is to investigate both the biomechanical feasibility of biceps transfer and the tendon excursion that results from transfer into the extensor digitorum communis (EDC) or flexor digitorum profundus (FDP) musculotendinous unit in both passive and active modes using anatomical data collected from fresh-frozen cadaver specimens.

\section{Methods}

\section{Surgical Technique}

Four fresh-frozen cadavers of the upper extremity including the lateral clavicle and scapula were used for this study. Demographic data of the cadavers is shown (Table 1). Each specimen was thawed to room temperature prior to dissection. Passive range of motion of the elbow, wrist, and digits was measured by physical exam and noted to be full in each specimen. No evidence of previous surgery or deformity was noted in any limb. Transfer of the distal biceps to the EDC was performed in two cadavers (specimens 1 and 2) and transfer to the FDP was performed in two separate cadavers (specimens 3 and 4). Following transfer, passive and active testing was performed on each of the specimens.

For transfer of the distal biceps tendon to the EDC, an extensile skin incision was made to simulate surgical exposure of the distal biceps tendon through a generous anterolateral approach to the elbow. The biceps tendon was identified along its course across the elbow into its insertion on the radial tuberosity along with the lacertus fibrosus. The lacertus fibrosus was released from its fascial attachment and incorporated into the bulk of the biceps tendon. The biceps tendon was released sharply from its radial insertion, retrieved from the wound, and positioned more superficially across the wrist extensors. Soft tissue adhesions into the distal arm were bluntly released. The distal portion of the incision was made over the outcropping muscles to the thumb, and followed proximally, identifying the EDC and extensor carpi radialis brevis 
(ECRB) tendons through the posterior Thompson interval. The musculotendinous portion of the EDC was exposed. The biceps was then transferred into the EDC tendons in a Pulvertaft weave. The extensor fascia was then closed over the transfer site, effectively creating a pulley through which the transfer acts. Some elbow flexion (usually 60 degrees) was required to ensure the biceps could be woven two to three times into the tendon without excessive tension.

For transfer to the FDP, dissection proceeded distally and ulnarly in two separate cadaver specimens. A McConnell-type approach was performed, identifying the flexor digitorum superficialis (FDS) and flexor carpi ulnaris (FCU) interval, and protecting the underlying ulnar artery and nerve. The FDP muscle was identified deep to the FDS and adjacent to the ulnar neurovascular bundle. The biceps muscle was tunneled deep to the FDS muscle belly and sutured into the FDP musculotendinous junction. Tunneling the tendon through the FDS muscle belly created a fulcrum for the transfer and prevented bowstringing of the tendon.

To facilitate measurement of tendon excursion, a metal marker was embedded into the donor tendon. For the FDP, a central tendon was selected just proximal to the carpal tunnel. The metal marker was sutured deep into the tendon. The marker was sutured into the EDC tendon in a similar fashion just proximal to extensor retinaculum. Two K-wires were placed $10 \mathrm{~mm}$ apart in the distal ulna to later allow for post-imaging scaling and measurement using Adobe Photoshop Elements 6.0.

A commercial hinged elbow external fixator (Orthofix International, Lewisville, TX, USA) was applied to the arm in the recommended fashion using fluoroscopic assistance. This allowed for ease in performing two observations: the effect of passive elbow flexion/extension on digital motion and the effect of active biceps loading on digital motion. 
Forearm positioning was accounted for by fixing the forearm in neutral rotation of the humeral-forearm axis using two 0.062 ” K-wires inserted from ulnar to radial across four cortices of the ulna and radius.

\section{Experimental Testing}

Following muscle transfer, fixation in forearm neutral prono-supination and wrist neutral flexion, and external fixator application, the elbow was taken through a passive range of motion within the allowable limits based on soft tissue tension. A goniometer was used to measure the position of the elbow in $15^{\circ}$ increments $\left(30,45,60,75,90^{\circ}\right)$ and fluoroscopic images were taken to indirectly measure tendon excursion by imaging the embedded metal marker. The effects of passive elbow movement on extensor tendon excursion are shown (Figure 1).

After passive testing, variable simulated active loading through the distal biceps was applied. With elbow flexion locked at $90^{\circ}$, the distal biceps tendon was loaded by weaving a high-tensile strength suture (USP \#5 Ethibond, Ethicon, Inc., Cincinnati, OH, USA) through the tendon unit, and tunneling it through the arm posteriorly in line with the biceps tendon fibers. The suture was strung over the edge of the table at a 90-degree angle along a pulley. The end of the suture was then tied to a one pound weight holder. The position of the metal marker without loading was noted using fluoroscopy. Loads of 1, 3, and 5 pounds were applied with fluoroscopic images taken after each load. Maximal contraction was then simulated by adding weight until no additional motion could be detected based on fluoroscopy. This amounted to greater than 20 pounds in each of the four specimens. The effects of active biceps loading on flexor tendon excursion are shown (Figure 2)

\section{Muscle Characteristics}


Measurement of biceps muscle physiologic cross-sectional area (PCSA) and muscle fiber length was performed after excursion and load testing. The transfer site was released and the entire biceps muscle removed from the anterior compartment of the arm and harvested proximally from its insertions on the coracoid and glenoid rim. Any attachments to the adjacent brachialis were released.

The PCSA of the biceps muscle was calculated by dividing the muscle volume by true fiber length. Determining muscle volume was performed by the water displacement method as described by An et al. ${ }^{2}$ The muscle was submersed in a full graduated beaker and the displaced water was measured using a pipette and recorded in cubic centimeters.

The true muscle fiber length was then determined by measuring the length of muscle between proximal and distal tendinous units as described by Brand et al. ${ }^{3}$

\section{Results}

Passive loading after transfer into the EDC is shown (Table 2). In each cadaver, a maximum passive excursion of $24 \mathrm{~mm}$ was obtained. Active loading of these same specimens showed maximum excursions of 23 and $24 \mathrm{~mm}$ (Table 3).

Passive loading after transfer into the FDP resulted in a maximum of 23 and $24 \mathrm{~mm}$ of excursion (Table 4). Active loading of these specimens resulted in maximum excursion of 24 $\mathrm{mm}$ in both (Table 5).

The properties of the cadaver specimens are shown along with the calculated physiological cross-sectional area (Table 6).

\section{Discussion}

Restoring finger function is an important goal for many patients with tetraplegia, Volkmann's contractures, or brachial plexopathies. This can prove challenging, and as the 
number of available motor units to transfer decreases, as is the case with higher levels of spinal cord injury, the options we can offer patients decrease.

Described methods of reestablishing finger flexion include tenodesis of FDP, transfer of extensor carpi radialis longus (ECRL) or pronator teres (PT) to FDP, and functional free gracilis transfer to medial epicondyle proximally and FDP distally. Methods for reestablishing finger extension include tenodesis of EDC and/or hand intrinsics, transfer of extensor pollicis longus (EPL) to EDC, and functional free gracilis transfer to the lateral epicondyle proximally and EDC distally.

Transfer of the biceps tendon has been utilized for different conditions. With its higher level of spinal cord innervation, biceps function may be present in tetraplegic patients lacking function of the forearm muscles or triceps. Indeed, biceps-to-triceps transfer has proved a valuable tool for reestablishing elbow extension in select patients with tetraplegia. ${ }^{4,5}$ In treating cerebral palsy, Manske et al. described detachment and rerouting of the biceps tendon in addition to percutaneous osteoclasis for fixed supination contracture. ${ }^{6}$ Goubier et al. described a technique using a fascia lata autograft to synergize biceps contraction with contraction of the FDP tendons without tendon transfer in patients with total brachial plexus palsy. ${ }^{7}$

We have shown biceps transfer to be feasible while obeying the basic principles of tendon transfer, allowing for approximately $24 \mathrm{~mm}$ of tendon excursion of the EDC and FDP tendons with the wrist immobilized and prono-supination in neutral. While our study controlled for wrist flexion/extension and forearm rotation, it should be noted that this transfer is likely to have additional effects in vivo on both wrist motion and forearm rotation as the distal biceps is a strong supinator, and the vector of biceps activity is significantly altered. 
In comparing the anatomic data of the biceps tendon, the PCSA in our study averaged 3.6 $\mathrm{cm}^{2}$ with some variability, due mainly to the volume of the specimens. Previous data from An et. al. compared well to our data, with a mean PCSA of $4.4 \mathrm{~cm}^{2}{ }^{2}$ The same authors also calculated the PCSA of the EDC and FDP muscles, which was not done in our study. Their results were 3.8 and $6.1 \mathrm{~cm}^{2}$, respectively. Considering the general work capacity of the individual muscles, the biceps matches well with the EDC, but is less ideal when comparing to the FDP. However, in absence of a more suitably matched donor some function may be preferable to none.

This tendon transfer has a highly select patient population, and would be contraindicated if there is concern for maintaining full active strength of supination of the forearm and/or flexion of the elbow. The brachialis and supinator muscles are innervated from a similar level as the biceps, so some ability to flex and supinate should remain after biceps transfer. Loss of function after distal biceps tendon transfer cannot be determined or approximated in our study but may mimic distal biceps tendon rupture. One would expect approximately $40 \%$ loss in forearm supination strength and 30\% loss of elbow flexion strength. ${ }^{8,9}$ Close attention to physical exam findings as well as a thorough discussion with the patient and family is warranted to temper patient expectations.

Finger motion depends on both passive position of the wrist as well as the synchronous activation and inactivation of muscle groups in the forearm. In surgical treatment of tetraplegia, it is common to combine tendon transfers, tenodesis, and arthrodesis to provide an optimal outcome. ${ }^{10}$ The excursions identified in this study are below the 5 and $7 \mathrm{~cm}$ provided in normal digit extensors and flexors. ${ }^{11}$ However, there is significant precedent for transferring tendons with less excursion than the native recipient target (e.g. FCR to finger extensors in radial nerve palsy). Often in tetraplegia, when options are limited, some function is much better than no 
function. It is also reasonable to expect that additional procedures may be necessary or useful with this tendon transfer to optimize restoration of function.

This study does have several shortcomings. The nature of cadaveric study may not represent the effects on live tissue or allow evaluation of the dynamic features in this tendon transfer. The pre-mortal state of each limb could not be determined, but most likely differs from the patients in which this transfer would be used and the limb of a "previously healthy" cadaver may not approximate the limb of a patient that may benefit from this transfer. Although there were only two cadavers in each transfer, the data was consistent between specimens.

Future outcome studies are warranted to correlate these theoretical and laboratory predictions into clinical results that confirm good finger function with this transfer and to quantify the degree of function lost from sacrificing the biceps. 


\section{References}

1. Beasley RW. Principles of tendon transfer. Orthop Clin North Am. 1970;1(2):433-438.

2. An KN, Hui FC, Morrey BF, Linscheid RL, Chao EY. Muscles across the elbow joint: a biomechanical analysis. J Biomechanics. 1981;14(10):659-669.

3. Brand PW, Beach RB, Thompson DE. Relative tension and potential excursion of muscles in the forearm and hand. J Hand Surg. 1981;6(3):209-219.

4. Friedenberg ZB. Transposition of the biceps brachii for triceps weakness. J Bone Joint Surg. 1954;36(3):656-658.

5. Kozin SH, D’Addesi L, Chafetz RS, Ashworth S, Mulcahey MJ. Biceps-to-triceps transfer for elbow extension in persons with tetraplegia. J Hand Surg Am. 2010;35(6):968-975.

6. Manske PR, McCarroll HR Jr, Hale R. Biceps tendon rerouting and percutaneous osteoclasis in the treatment of supination deformity in obstetrical palsy. J Hand Surg Am. 1980;5(2):153-159.

7. Goubier JN, Teboul F. Restoration of active fingers flexion with tensor fascia lata transfer in total brachial plexus palsy. Tech Hand Up Extrem Surg. 2009;13(1):1-3.

8. Baker BE, Bierwagen D.: Rupture of the distal tendon of the biceps brachii. Operative versus non-operative treatment. J Bone Joint Surg Am. 1985;(67)3:414-417.

9. Morrey BF, Askew LJ, An KN, Dobyns JH. Rupture of the distal tendon of the biceps brachii. A biomechanical study. J Bone Joint Surg Am. 1985;(67)3:418-421.

10. Fridén J, Gohritz A. Tetraplegia management update. J Hand Surg Am. 2015;40(12):2489-2500.

11. Boyes JH. Bunnell's Surgery of the Hand. 5th ed. Philadelphia, PA: JB Lippincott; 1970. 
Biceps Transfer for Extrinsic Finger Function

\begin{tabular}{|ccccc|}
\hline Specimen & Sex & Age & Weight $(\mathrm{lb})$ & BMI \\
\hline 1 & Male & 78 & 112 & 17 \\
2 & Male & 63 & 130 & 22 \\
3 & Male & 70 & 244 & 36 \\
4 & Male & 34 & 141 & 20 \\
\hline Mean & & 61.25 & 156.75 & 23.75 \\
\hline
\end{tabular}

Table 1. Cadaver specimen demographics. 
Biceps Transfer for Extrinsic Finger Function

\begin{tabular}{|c|cc|}
\hline & \multicolumn{2}{|c|}{ Extensor tendon excursion $(\mathrm{mm})$} \\
\hline Elbow Flexion & Specimen 1 & Specimen 2 \\
\hline $90^{\circ}$ & - & - \\
$75^{\circ}$ & 12 & 14 \\
$60^{\circ}$ & 20 & 20 \\
$45^{\circ}$ & 24 & 22 \\
$30^{\circ}$ & $\mathrm{n} / \mathrm{a}$ & 24 \\
\hline
\end{tabular}

Table 2. Extensor tendon excursion at the distal radius with passive elbow range of motion. 
Biceps Transfer for Extrinsic Finger Function

\begin{tabular}{|c|cc|}
\hline & \multicolumn{2}{|c|}{ Extensor tendon excursion $(\mathrm{mm})$} \\
\hline Load (lbs) & Specimen 1 & Specimen 2 \\
\hline 0 & - & - \\
1 & 2 & 2 \\
3 & 10 & 10 \\
5 & 18 & 20 \\
$>20$ & 23 & 24 \\
\hline
\end{tabular}

Table 3. Extensor tendon excursion at the distal radius with variable direct loading of the transferred biceps tendon. 
Biceps Transfer for Extrinsic Finger Function

\begin{tabular}{|c|cc|}
\hline & \multicolumn{2}{|c|}{ Flexor tendon excursion $(\mathrm{mm})$} \\
\hline Elbow Flexion & Specimen 3 & Specimen 4 \\
\hline $90^{\circ}$ & - & - \\
$75^{\circ}$ & 10 & 8 \\
$60^{\circ}$ & 15 & 16 \\
$45^{\circ}$ & 20 & 24 \\
$30^{\circ}$ & 23 & $\mathrm{n} / \mathrm{a}$ \\
\hline
\end{tabular}

Table 4. Flexor tendon excursion at the carpus with passive elbow range of motion. 
Biceps Transfer for Extrinsic Finger Function

\begin{tabular}{|c|cc|}
\hline & \multicolumn{2}{|c|}{ Flexor tendon excursion $(\mathrm{mm})$} \\
\hline Load (lbs) & Specimen 3 & Specimen 4 \\
\hline 0 & - & - \\
1 & 3 & 4 \\
3 & 8 & 8 \\
5 & 18 & 20 \\
$>20$ & 24 & 24 \\
\hline
\end{tabular}

Table 5. Flexor tendon excursion at the carpus with variable direct loading of the transferred biceps tendon. 
Biceps Transfer for Extrinsic Finger Function

\begin{tabular}{|cccc|}
\hline Specimen & Volume $\left(\mathrm{cm}^{2}\right)$ & Fiber length $(\mathrm{cm})$ & PCSA of biceps $\left(\mathrm{cm}^{2}\right)$ \\
\hline 1 & 32.2 & 18.8 & 1.7 \\
2 & 52.2 & 18.7 & 2.8 \\
3 & 122.4 & 18.4 & 6.7 \\
4 & 58.6 & 17.9 & 3.3 \\
\hline Mean & 66.4 & 18.5 & 3.6 \\
\hline
\end{tabular}

Table 6. Cadaveric biceps muscle properties including measured volume and fiber length, and calculated physiologic cross-sectional area (PSCA). 
Figure 1. The effects of passive biceps loading via elbow extension on excursion of extensor digitorum communis (EDC) at the distal radius. A) The resting position of the fingers with the elbow flexed to $90^{\circ}$. B) Finger extension demonstrated with elbow extension to $30^{\circ}$. Note the excursion of the metal marker implanted in the EDC tendon (circled in red). Figure 2. The effects of direct biceps loading on excursion of flexor digitorum profundus (FDP) at the distal radius with the elbow fixed at $90^{\circ}$. A) The resting position of the fingers with no loading. B) Finger flexion demonstrated with maximum loading of the biceps tendon (>20 lbs.). Note the excursion of the metal marker implanted in the FDP tendon (circled in red). 